\title{
RIGID 3-DIMENSIONAL COMPACTA WHOSE SQUARES ARE MANIFOLDS
}

\author{
FREDRIC D. ANCEL, ${ }^{1}$ PAUL F. DUVALL AND S. SINGH
}

\begin{abstract}
A space is rigid if its only self-homeomorphism is the identity. In response to a question of Jan van Mill, Ancel and Singh have given examples of rigid $n$-dimensional compacta, for each $n \geqslant 4$, whose squares are manifolds. We construct a rigid 3-dimensional compactum whose square is the manifold $S^{3} \times S^{3}$. In fact, we construct uncountably many topologically distinct compacta with these properties.
\end{abstract}

1. Introduction. A space is rigid if it does not have any self-homeomorphism other than the identity. In response to a question of Arhangel'skii [2], Jan van Mill constructed a rigid infinite dimensional compactum $X$ whose square $X \times X$ is homogeneous [7]. This result led van Mill to raise the following question (see [7]): Does there exist a rigid finite dimensional compactum whose square is homogeneous or perhaps even a (Lie) group? An affirmative answer to this question is provided in [1], where the following result is proved. For each integer $n \geqslant 4$, there exists a rigid $n$-dimensional compactum $X$ such that $X \times X$ is a closed $2 n$-manifold (and hence homogeneous). Moreover $X \times X$ can be chosen to be a Lie group. There are uncountably many topologically distinct compacta $X$ with these properties.

The purpose of this note is to prove a similar result in the dimension, $n=3$, which eluded [1]. As in [7] and [1], each of the spaces constructed here is rigid because it contains a countable dense set of points whose individual point complements are topologically distinct. Moreover, the point complements are distinguished by their proper homotopy type.

We wish to thank Thomas Thickstun for many helpful conversations.

\section{The main result.}

THEOREM. There is a null cell-like decomposition $G$ of $S^{3}$ such that $S^{3} / G$ is a rigid 3-dimensional compactum and $S^{3} / G \times S^{3} / G$ is homeomorphic to (the Lie group) $S^{3} \times S^{3}$.

Proof. We begin by recalling the construction in [6]. $P$ is an uncountable index set whose elements are certain infinite sequences of primes. For each $p \in P, W_{p}$ is a

Received by the editors October 28, 1982.

1980 Mathematics Subject Classification. Primary 54G20, 57P99; Secondary 54B15, 55M15.

Key words and phrases. Cell-like compactum, homogeneous, manifold, generalized 3-manifold, cell-like decomposition.

'Partially supported by N.S.F. Grant No. MCS 8103420. 
contractible open subset of $R^{3}=S^{3}-\{\infty\}$ such that for distinct elements $p$ and $q$ of $P, W_{p}, W_{q}$ and $R^{3}$ are topologically distinct. Furthermore, for each $p \in P$, $W_{p}=\cup_{i=0}^{\infty} T_{i}$ such that for each $i \geqslant 0$ :

(1) $T_{i}$ and $U_{i}=\mathrm{cl}\left(S^{3}-T_{i}\right)$ are solid tori in $S^{3}$,

(2) $T_{i} \subset \operatorname{int}\left(T_{i+1}\right)$, and

(3) $T_{i}$ contracts to a point in $\operatorname{int}\left(T_{i+1}\right)$.

$\left(\left\{T_{i}\right\}\right.$ and $\left\{U_{i}\right\}$ vary with the choice of $p \in P$.) We shall actually need to know that for distinct elements $p$ and $q$ of $P, W_{p}, W_{q}$ and $R^{3}$ have distinct proper homotopy types; this follows from Corollary (2.6) of [4].

For each $p \in P$, let $C_{p}=S^{3}-W_{p}$. The nondegenerate elements of the decomposition $G$ are chosen from among the $C_{p}$ 's. So we must verify that each $C_{p}$ is cell-like. Let $p \in P$. Since $C_{p}=\cap_{i=0}^{\infty} U_{i}$, it suffices to show that $U_{i+1}$ cóntracts to a point in $U_{i}$ for each $i \geqslant 0$. Let $i \geqslant 0$. Since $T_{i}$ contracts to a point in $\operatorname{int}\left(T_{i+1}\right)$, the simple closed curve core $J$ of $T_{i}$ is null-homologous in the complement of the simple closed curve core $K$ of $U_{i+1}$. From the symmetry of linking number, it follows that $K$ is null-homologous in $S^{3}-J$. Since $\pi_{1}\left(S^{3}-J\right) \approx \pi_{1}\left(S^{3}-U_{i}\right)=\pi_{1}\left(\right.$ int $\left.T_{i}\right) \approx \mathbf{Z}$, then the abelianization homomorphism $\pi_{1}\left(S^{3}-J\right) \rightarrow H_{1}\left(S^{3}-J\right)$ is an isomorphism. Therefore $K$ is null-homotopic in $S^{3}-J$. It follows that $U_{i+1}$ contracts to a point in $S^{3}-T_{i}=\operatorname{int}\left(U_{i}\right)$.

To specify the nondegenerate elements of $G$, choose a sequence $p(1), p(2), \ldots$ of distinct elements of $P$. Reposition $C_{p(1)}, C_{p(2)}, \ldots$ in $S^{3}$ (by homeomorphisms of $S^{3}$ ) so that

(1) they are disjoint,

(2) their union is dense in $S^{3}$, and

(3) $\operatorname{diam} C_{p(i)}<1 / i$.

Let $G$ be the null cell-like decomposition of $S^{3}$ whose nondegenerate elements are $C_{p(1)}, C_{p(2)}, \ldots$

A homeomorphism from the square $S^{3} / G \times S^{3} / G$ to $S^{3} \times S^{3}$ is provided by [3].

It remains to show that $S^{3} / G$ is 3 dimensional and rigid. Let $\pi: S^{3} \rightarrow S^{3} / G$ denote the quotient map, and let $x_{i}=\pi\left(C_{p(i)}\right)$ for each $i \geqslant 1$. Since the singular set $\left\{x_{i}: i \geqslant 1\right\}$ of $\pi$ is countable, then $\pi$ preserves dimension, and $\pi \mid \pi^{-1} V: \pi^{-1} V \rightarrow V$ is a proper homotopy equivalence for each open subset $V$ of $S^{3} / G$ (cf. [5]). Hence $\operatorname{dim} S^{3} / G=3$. Since for $i \neq j$ and $y \in S^{3}-\cup_{k=1}^{\infty} C_{p(k)}, W_{p(i)}=S^{3}-C_{p(i)}, W_{p(j)}$ $=S^{3}-C_{p(j)}$ and $S^{3}-\{y\}$ have distinct proper homotopy types, then so do their images under $\pi$. Thus for $i \neq j$ and $z \in S^{3} / G-\left\{x_{i}: i \geqslant 1\right\}, S^{3} / G-\left\{x_{i}\right\}, S^{3} / G-$ $\left\{x_{j}\right\}$ and $S^{3} / G-\{z\}$ have distinct proper homotopy types. Therefore, no $x_{i}$ can be moved by any homeomorphism of $S^{3} / G$. Since $\left\{x_{i}: i \geqslant 1\right\}$ is a dense subset of $S^{3} / G$, we conclude that $S^{3} / G$ is rigid.

COROLLARY. There are uncountably many distinct rigid 3-dimensional compacta whose squares are homeomorphic to (the Lie group) $S^{3} \times S^{3}$.

The corollary follows immediately from the proof of the theorem. 


\section{REFERENCES}

1. F. D. Ancel and S. Singh, Rigid finite dimensional compacta whose squares are manifolds, Proc. Amer. Math. Soc. 87 (1982), 342-346.

2. A. V. Arhangel'skii, Structures and classification of topological spaces and cardinal invariants, Russian Math. Surveys 33 (1978), 33-96.

3. C. D. Bass, Some products of topological spaces are manifolds, Proc. Amer. Math. Soc. 81 (1981), 641-646.

4. E. M. Brown, Contractible 3-manifolds of finite genus at infinity, Trans. Amer. Math. Soc. 245 (1978), $503-514$.

5. R. C. Lacher, Cell-like mappings and their generalizations, Bull. Amer. Math. Soc. 83 (1977), 495-552.

6. D. R. McMillan, Jr., Some contractible open 3-manifolds, Trans. Amer. Math. Soc. 102 (1962), 373-382.

7. J. van Mill, $A$ rigid space $X$ for which $X \times X$ is homogeneous; an application of infinite dimensional topology, Proc. Amer. Math. Soc. 83 (1981), 597-600.

Department of Mathematics, University of OKLahoma, Norman, OKLahoma 73019

Department of Mathematics, OKlahoma State University, Stillwater, OKlahoma 74078

Department of Mathematics, Southwest TeXas State University, San Marcos, TeXas 78666 